\title{
ASSISTÊNCIA DE ENFERMAGEM EM URGÊNCIA E EMERGÊNCIA OBSTETRIICA: UM ESTUDO BIBLIOMÉTRICO
}

\author{
NURSING ASSISTANCE IN EMERGENCY OBSTETRICS AND EMERGENCY: A BIBLIOME- \\ TRIC STUDY
}

\author{
Leonardo Magela Lopes Matoso ${ }^{\mathbf{a}^{*}}$, Valéria Antônia de Lima ${ }^{b^{* *}}$, \\ leonardo.1.matoso@gmail.coma, valerialima2011@live.comb \\ Universidade do Estado do Rio Grande do Norte (Uern)*, Faculdade Unyleya ${ }^{* *}$
}

Data de Submissão: 19/02/2019 Data de Aceite:21/10/2019

\section{RESUMO}

Introdução: $O$ período gestacional é um processo fisiológico e natural no qual produz uma gama de modificações locais e sistêmicas no organismo feminino. No entanto, às vezes algumas distorcias acontecem durante este processo ocasionando as urgências e emergências. Objetivo: Este estudo objetivou descrever a produção científica da enfermagem sobre as urgências e emergências obstétricas. Materiais e Métodos: Realizou-se estado da arte, de natureza descritiva, desenvolvida a partir de uma análise bibliométrica. Foram trabalhadas duas bases de dados, a saber: Biblioteca Virtual em Saúde (BVS) e a Biblioteca Digital Brasileira de Teses e Dissertações (BDTD). A busca aconteceu por meio das palavras urgências, emergência e obstetrícia. Optou-se por trabalhar textos completos e dos últimos 10 anos, na língua portuguesa e espanhola. Aplicados critérios de inclusão e analisados estudos pertinentes para a área elencou-se seis estudos, formando assim, o corpus deste trabalho. Resultados: Infere-se que a assistência de enfermagem se encontra alicerçada no processo de enfermagem, com destaque na realização da triagem; serviços burocráticos, na monitorização dos sinais vitais, na administração de medicamentos e controle de equipamentos. Os resultados revelaram a existência de poucos estudos sobre as urgências e emergências obstétricas. Conclusão: Desse modo, sugere-se que mais estudos sejam realizados sobre essa temática, uma vez que é de extrema necessidade apreender sobre a condução assistencial, perfil profissional e a percepção das gestantes nas nuances que circundam as urgências e emergências obstétricas.

Palavras-chave: Urgências; emergência; obstetrícia; enfermagem.

\section{ABSTRACT}

Introduction: The gestational period is a physiological and natural process in which it produces a range of local and systemic changes in the female organism. However, sometimes some distortions occur during this process leading to emergencies and emergencies. Objective: This study aimed to describe the scientific production of nursing on emergency and obstetric emergencies. Materials and Methods: A state of the art, of a descriptive nature, was developed from a bibliometric analysis. Two databases were developed, namely: Virtual Health Library (VHL) and the Brazilian Digital Library of Theses and Dissertations (BDTD). The search took place through the words urgency, emergency and obstetrics. We chose to work full texts and of the last 10 years, in Portuguese and Spanish. Applied inclusion criteria and analyzed relevant studies for the area were listed six studies, thus forming the corpus of this work. Results: It is inferred that nursing care is based on the nursing process, with emphasis on the triage; bureaucratic services, vital signs monitoring, drug administration and equipment control. The results revealed the existence of few studies on emergency and obstetric emergencies. Conclusion: Therefore, it is suggested that more studies be carried out on this topic, since it is extremely necessary to learn about care management, professional profile and the perception of pregnant women in the nuances that surround emergency and obstetric emergencies.

Keywords: Urgency; emergency; obstetrics; nursing 


\section{Introdução}

A gestação é um processo fisiológico e natural no qual produz uma gama de modificações locais e sistêmicas no organismo feminino. Algumas vezes essas modificações podem acarretar quadros patológicos, tornando o processo reprodutivo uma situação de alto risco tanto para a mãe como para o concepto. Para que isso não aconteça as gestantes necessitam de uma assistência pré-natal humanizada, equânime e mais específica; com uma maior qualidade, para rastrear e eliminar possíveis comorbidades, reduzindo assim, as altas taxas de mortalidade materna e infantil ${ }^{1}$.

Autores $^{2,3}$ defendem que a maioria das gestações evolui sem nenhum tipo de intercorrências, porém durante o seu transcorrer algumas complicações que levam a mortalidade e morbidade podem ameaçar a vida materna e do concepto, configurando assim, em uma situação de urgência e emergência obstétrica, necessitando de uma intervenção adequada, imediata e em determinadas situações, a interrupção da gestação.

Os serviços de urgência e emergência visam promover maior agilidade aos atendimentos através da realização de uma triagem, análise e avaliação dos pacientes, levando em consideração os riscos e seu grau de vulnerabilidade. Com isso, os serviços de urgência e emergência tem prioridade de atendimento as pessoas que apresentam casos mais graves e, nessa hora, não faz diferença a ordem de chegada 4 .

Diante do exposto, é valido ressaltar que existem vários fatores de riscos na gestação. Estes fatores de riscos podem agir de forma isolada ou conjunta, dentre os quais se podem destacar as condições sócio demográficas, extremos de idade, hipertensão arterial prévia, diabetes, antecedentes gestacionais desfavoráveis ${ }^{5}$. Estes fatores podem causar complicações, sendo estas manifestadas no decorrer da gravidez ou durante o trabalho de parto.

Denota-se que as mortalidades maternas e perinatais constituem um problema de saúde pública, partindo do pressuposto que grande parte das complicações e óbitos que surgem durante o ciclo gravídico-puerperal são preveníveis. Desse modo, caracteriza-se então como urgência e emergência obstétrica uma situação que coloque em risco a vida da gestante e do feto, necessitando de um cuidado imediato de toda a equipe de saúde para reverter o quadro de perigo em que ambos se encontram ${ }^{6}$.

Neste interim, se faz necessário à qualificação do serviço de atenção primária e o aumento do quantitativo de profissionais habilitados $\mathrm{e}$ especializados na área de urgência e emergência direcionados a atenção à saúde obstétrica, para que seja possível realizar uma assistência qualificada, eliminando assim, possíveis complicações para a mulher e recém-nascido.

Dentre os profissionais da saúde responsáveis por essa assistência nos setores de urgência e emergência, tem-se o profissional de enfermagem. O enfermeiro, embasado por seu saber-fazer, tem o papel de prestar uma assistência integral e humanizada as mulheres que apresentam distorcias gravídicas e que adentram o serviço de urgência e emergência.

Destarte, pontifica-se como questão norteadora que impulsionou a realização deste estudo: qual o panorama da produção acadêmica de enfermagem sobre as urgências e emergências que envolvem gestantes?

Este estudo se justifica pelo interesse pessoal em aprofundar tal temática, uma vez, que possui relevância social e profissional. Além disso, enquanto enfermeiro, observa-se corriqueiramente na prática assistencial, lacunas acerca da assistência materno-infantil nas urgências e emergências. Com isso, a concatenação desta pesquisa pode auxiliar profissionais da saúde a atuarem nas distorcias obstétricas inferindo uma assistência mais humanizada e resolutiva.

Sendo assim, o presente estudo possui relevância por concatenar os achados literários acerca da assistência de enfermagem as urgências e emergências obstétricas em um único documento. Espera-se com isso incitar reflexões sobre a importância da educação continuada em enfermagem e sobre a construção de novas estratégias terapêuticas diante das eventuais distorcias grávidas e puerperais. Uma vez que se apreende que é a construção teórica, uma ferramenta capaz de atuar como mobilizadora e a transformadora da realidade assistencial.

Diante do exposto, este estudo tem como objetivo geral descrever a produção científica de 
enfermagem sobre as urgências e emergências obstétricas. É, portanto, um compilado teórico e bibliométrico sobre o estado da arte que circunda as urgências e emergências obstétricas.

\section{Metodologia}

Trata-se de uma revisão literária sobre o estado da arte, de natureza descritiva, desenvolvida a partir de uma análise bibliométrica, cuja unidade de análise constituiu-se por artigos, teses e dissertações publicados no Brasil relacionadas à temática: assistência de enfermagem as urgências e emergências obstétricas.

Os estudos bibliométricos são usados para quantificar os processos de comunicação escrita e o uso de indicadores bibliométricos para medir a produção científica. Os indicadores bibliométricos utilizam dados sobre a produção de artigos científicos e o registro de patentes, para avaliar os resultados dos investimentos em pesquisas e para responder aos questionamentos sobre o impacto das pesquisas na comunidade científica, ou seja, esse estudo busca analisar essas várias dimensões, buscando conhecer o que já foi debatido e contribuir para o direcionamento das futuras produções acadêmicas ${ }^{7}$.

Como base de coleta de dados para realização deste estudo de caráter bibliométrico, utilizou-se a Biblioteca Virtual em Saúde (BVS) e a Biblioteca Digital Brasileira de Teses e Dissertações (BDTD), disponíveis nos respectivos links <brasil.bvs. br/>, <http://bdtd.ibict.br/vufind/>. Essas bases de dados são de cunho descentralizado, onde agrega uma gama de estudos nacionais sobre os mais variados temas. É um espaço de integração de fontes de informação cientifica que promove a democratização e ampliação do acesso à informação científica no Brasil.

Foram utilizados como critério de seleção, a saber: artigos completos, teses ou dissertações disponíveis nos bancos de dados que mencionarem os descritores ou expressões de busca: "Urgência" OR "Emergência" AND "Obstetrícia” publicadas entre os últimos dez anos. Foram incluídas nos bancos de dados os artigos, teses e dissertações baseadas na temática, em lingual portuguesa e espanhola, sejam isoladas ou em conjunto com outras metodologias ou trabalhos que disponibilizassem os dados necessários, como ano de publicação, instituição, tipo de trabalho e trabalhos que abordassem diretamente a temática.

Depois desta seleção os materiais foram lidos criteriosamente dando-se início ao preenchimento de um instrumento de análise bibliométrica. $\mathrm{O}$ instrumento permitiu a sistematização dos dados em forma descritiva. Os dados foram tabulados no software da Microsoft Excel 2011 na qual foram extraídos tabelas e gráficos, sendo possível retirar duas estruturas gerais de informações para análise:

1) Totalidades das pesquisas encontradas no banco coletaram-se as seguintes informações: número total de resumos, idioma, tipo de trabalho (tese/dissertação) e ano de publicação;

2) Tipos de assistência em enfermagem dispensados em casos de urgência e emergência obstetrícia.

Assim, de posse do instrumento devidamente preenchido, fez-se a análise estatística descritiva, com registro das frequências das informações obtidas, onde foram confrontadas e correlacionadas as teses e as dissertações selecionadas, visando compreender as vertentes e sendo possível responder ao objetivo proposto.

É válido ressaltar que a aprovação desta pesquisa por um Comitê de Ética em Pesquisas com Seres Humanos não se mostrou necessário, visto que o estudo é de caráter bibliométrico, sem envolvimento direto de seres humanos como sujeitos da pesquisa, e os dados disponíveis no banco são de domínio público. Portanto, as citações e direitos autorais utilizados neste estudo foram mantidos, levando à risca as normativas da Associação Brasileira de Normas Técnicas (ABNT).

\section{Resultados}

Os resultados inerentes a coleta de dados deste estudo foram realizados em duas bases de dados distintas e concatenadas para análise. A investigação inicial mostrou a escassez literária acerca da temática proposta, este fato será sintetizado e discutido ao longo deste estudo. 
$\mathrm{Na}$ BVS, foram utilizados os descritores "Urgência" OR "Emergência" AND "Obstetrícia" associados com os operadores booleanos. Quando esses três descritores foram associados os resultados revelaram 522 artigos disponíveis. Quando foi aplicado o critério de inclusão "texto completo", os números de artigos foram reduzidos para 220. Ademais, foram aplicados os critérios de "idioma na língua portuguesa e espanhola", bem como documentos nos "últimos 10 anos", assim, o número final decaiu para 22 artigos, onde estes foram extraídos para leitura flutuante. Após leitura foram selecionados seis documentos (artigos e dissertação) [(Quadro 1)].

Quadro 1: Material selecionado para compôs este estudo

\begin{tabular}{|c|c|c|c|c|}
\hline Ano & Autor & Titulo & Documento & Objetivo \\
\hline 2009 & $\begin{array}{l}\text { González } \\
\text { MEM, Fabero } \\
\text { OJN, Reyna } \\
\text { JCR }\end{array}$ & $\begin{array}{c}\text { Placenta prévia e urgências } \\
\text { no serviço obstétrico: um } \\
\text { estudo de caso }\end{array}$ & Artigo & $\begin{array}{l}\text { Conduta obstétrica nos casos de } \\
\text { placenta prévia em emergências. }\end{array}$ \\
\hline 2011 & $\begin{array}{l}\text { Oyarzun E, } \\
\text { Kusanovic PJ }\end{array}$ & Urgências em obstetrícia & Artigo & $\begin{array}{c}\text { Assistência em saúde acerca das } \\
\text { urgências obstétricas. }\end{array}$ \\
\hline 2014 & $\begin{array}{l}\text { Nomura RMY, } \\
\text { et al. }\end{array}$ & $\begin{array}{l}\text { Validação de questionário } \\
\text { para avaliar a vivência } \\
\text { e autoconfiança nas } \\
\text { emergências na assistência } \\
\text { ao parto vaginal }\end{array}$ & Artigo & $\begin{array}{c}\text { Validar questionário para } \\
\text { conhecer e descrever a percepção } \\
\text { dos médicos especialistas em } \\
\text { ginecologia e obstetrícia quanto } \\
\text { à vivência e autoconfiança no } \\
\text { atendimento de emergências no } \\
\text { parto vaginal. }\end{array}$ \\
\hline 2016 & $\begin{array}{l}\text { Ataide MM, } \\
\quad \text { et al. }\end{array}$ & $\begin{array}{c}\text { Exame obstétrico realizado } \\
\text { pela } \\
\text { enfermeira: da teoria à } \\
\text { prática }\end{array}$ & Artigo & $\begin{array}{l}\text { Analisar o conhecimento e a } \\
\text { prática da enfermeira acerca do } \\
\text { exame físico obstétrico. }\end{array}$ \\
\hline 2016 & $\begin{array}{l}\text { Brilhante AF, } \\
\text { et al. }\end{array}$ & $\begin{array}{l}\text { Implementação do protocolo } \\
\text { de acolhimento com } \\
\text { classificação de risco em } \\
\text { uma emergência } \\
\text { obstétrica }\end{array}$ & Artigo & $\begin{array}{c}\text { Avaliar a implementação do } \\
\text { Acolhimento com Classificação } \\
\text { de Risco. }\end{array}$ \\
\hline 2016 & Santos JKS & $\begin{array}{l}\text { Saúde da mulher: motivos } \\
\text { de atendimento no serviço } \\
\text { de urgência obstétrica às } \\
\text { gestantes residentes nas } \\
\text { áreas de atuação das ESF } \\
\text { no setor habitacional do sol } \\
\text { nascente, Ceilândia - DF, } \\
\text { 2014-2015. }\end{array}$ & Dissertação & $\begin{array}{l}\text { Avaliar os motivos de } \\
\text { atendimento no serviço de } \\
\text { urgência obstétrica às gestantes } \\
\text { residentes nas áreas de atuação } \\
\text { das UBS das equipes de ESF } \\
\text { no Setor Habitacional do Sol } \\
\text { Nascente (SHSN), Ceilândia/DF. }\end{array}$ \\
\hline
\end{tabular}

Fonte: dados da pesquisa, 2018 
No que se refere a coleta de dados na BDTD, foram utilizadas as expressões de busca "Urgência", "Emergência" e "Obstetrícia" e foram encontradas 18 teses/dissertações. No entanto, quando foram aplicados os critérios de seleção, levando em conta todos os critérios foi extraído e selecionado apenas uma dissertação de mestrado. Assim, o corpus deste estudo foram seis documentos (cinco artigos e uma dissertação) que abordavam a temática proposta.
Dos seis documentos elencados para compor este estudo, o principal veículo de divulgação encontrada na amostra analisada foi de artigos, representando 83\%. A Tabela 1 apresenta a distribuição dos documentos com base nos descritores, banco de dados, resultados e número de documentos extraídos com base nos descritores associados.

Tabela 1: Descrição sintética dos documentos com base nos descritores, banco de dados, resultados e número de documentos extraídos para este estudo

\begin{tabular}{ccccc}
\hline Descritores & Banco de Dados & Resultados & Extraídos & Selecionados \\
\hline $\begin{array}{c}\text { Urgência OR Emergência AND } \\
\begin{array}{c}\text { Obstetrícia } \\
\text { Urgência, Emergência e } \\
\text { Obstetrícia }\end{array}\end{array}$ & BVS & 522 & 22 & 5 \\
\multicolumn{1}{c}{ Total } & BDTD & 18 & 1 & 1 \\
\hline
\end{tabular}

Fonte: dados da pesquisa, 2018

Em relação ao idioma de publicação, $50 \%$ dos artigos foram publicados em espanhol, 33,4\% de artigos em português e $16,6 \%$ refere-se à dissertação em língua portuguesa (Ilustração 1).

Ilustração 1: Descrição sintética dos documentos com relação ao idioma

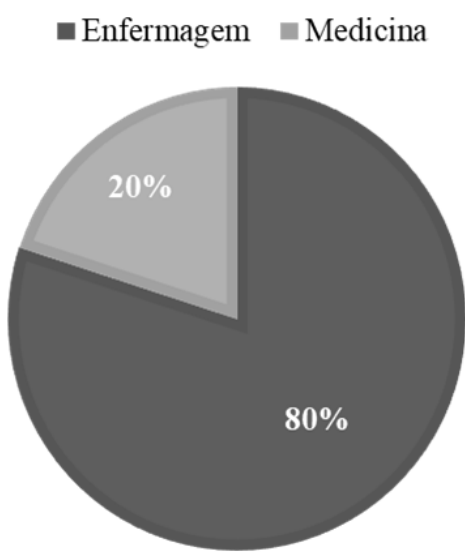

Fonte: dados da pesquisa, 2018

No que tange a área de concentração, os resultados apontaram que $80 \%$ dos estudos fazem parte da grande área Enfermagem e 20\% de Medicina; onde todos os estudos foram de cunho quantitativa (Ilustração 2).
Ilustração 2: Descrição sintética dos documentos com relação ao idioma

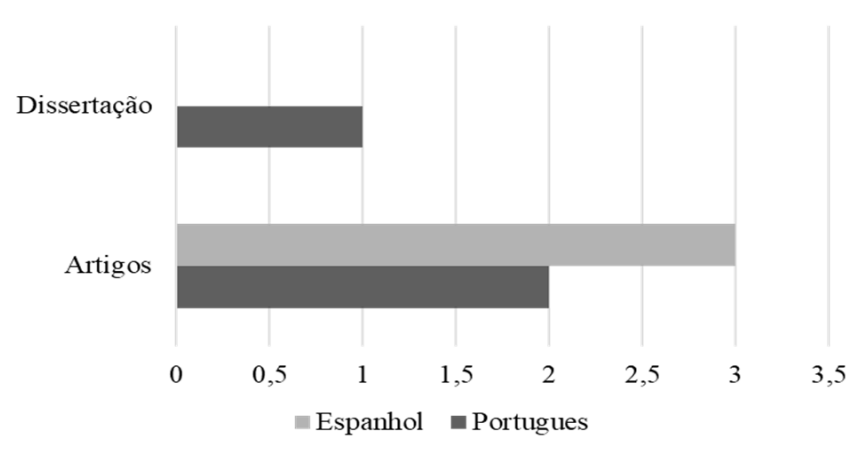

Fonte: dados da pesquisa, 2018

É importante deixar claro que mesmo os artigos sendo vinculados hegemonicamente na Enfermagem, isso não indica necessariamente que a produção real seja insignificante em outras áreas, uma vez que os critérios de seleção buscavam identificar a produção cientifica apenas na enfermagem, sobre a assistência que cabia ao enfermeiro desenvolvê-la.

No tocante às instituições formadoras nos quais os pesquisadores/autores estavam vinculados durante a condução da pesquisa 16,6\% encontrase na região Sudeste, Nordeste, Centro-Oeste e 
Sul; e 33,6 se referem a região Metropolitan, do Chile. Esses dados podem ser visualizados na tabela Tabela 2.

Tabela 2: Descrição dos artigos e dissertações com base nas instituições e região

\begin{tabular}{lcccc}
\hline \multicolumn{1}{c}{ Instituição } & Sigla & Região & N & \% \\
\hline Universidade Federal de Alagoas & UFAL & Sudeste & 1 & 16,6 \\
Universidade Federal do Ceará & UFC & Nordeste & 1 & 16,6 \\
Universidade de Brasília & UB & Centro-Oeste & 1 & 16,6 \\
Pontificia Universidad Católica de Chile & UCC & Metropolitan & 2 & 33,6 \\
Escola Paulista de Medicina da U.F São Paulo & UNIFESP & Sul & 1 & 16,6 \\
\hline Total & & & $\mathbf{6}$ & $\mathbf{1 0 0}$ \\
\hline
\end{tabular}

Fonte: dados da pesquisa, 2018.

Com relação ao ano de publicação $50 \%$ foram publicados em $2016 ; 16,6 \%$ em $2014 ; 16,6 \%$ em 2011 e 16,6\% em 2009. Os estudos revelaram que a assistência de enfermagem diante das urgências e emergências obstétricas recaem sobre a triagem (acolhimento); a monitorização dos sinais vitais; o controle dos drenos, cateteres, balanço hídrico, homeostase debito cardíaco e urinário; administração de medicamentos conforme protocolo hospitalar e/ou conduta médica; e serviços burocráticos, como regulação. A maioria destas condutas foram realizadas mediante algumas intercorrências obstétricas, como por exemplo sangramento, hipertensão arterial elevada, convulsão, pré-eclampsia e síndrome Hellp, o que se configuram em sua grande maioria como doenças hipertensivas específicas da gestação (DHEG). A Ilustração 3 aponta esses achados de forma mais categórica, relevando o número de vezes que essas condutas apareceram nos artigos/dissertação.

Ilustração 3: Assistências de enfermagem diante das urgências e emergências obstétricas de acordo com o com a frequência de discussão dos estudos.

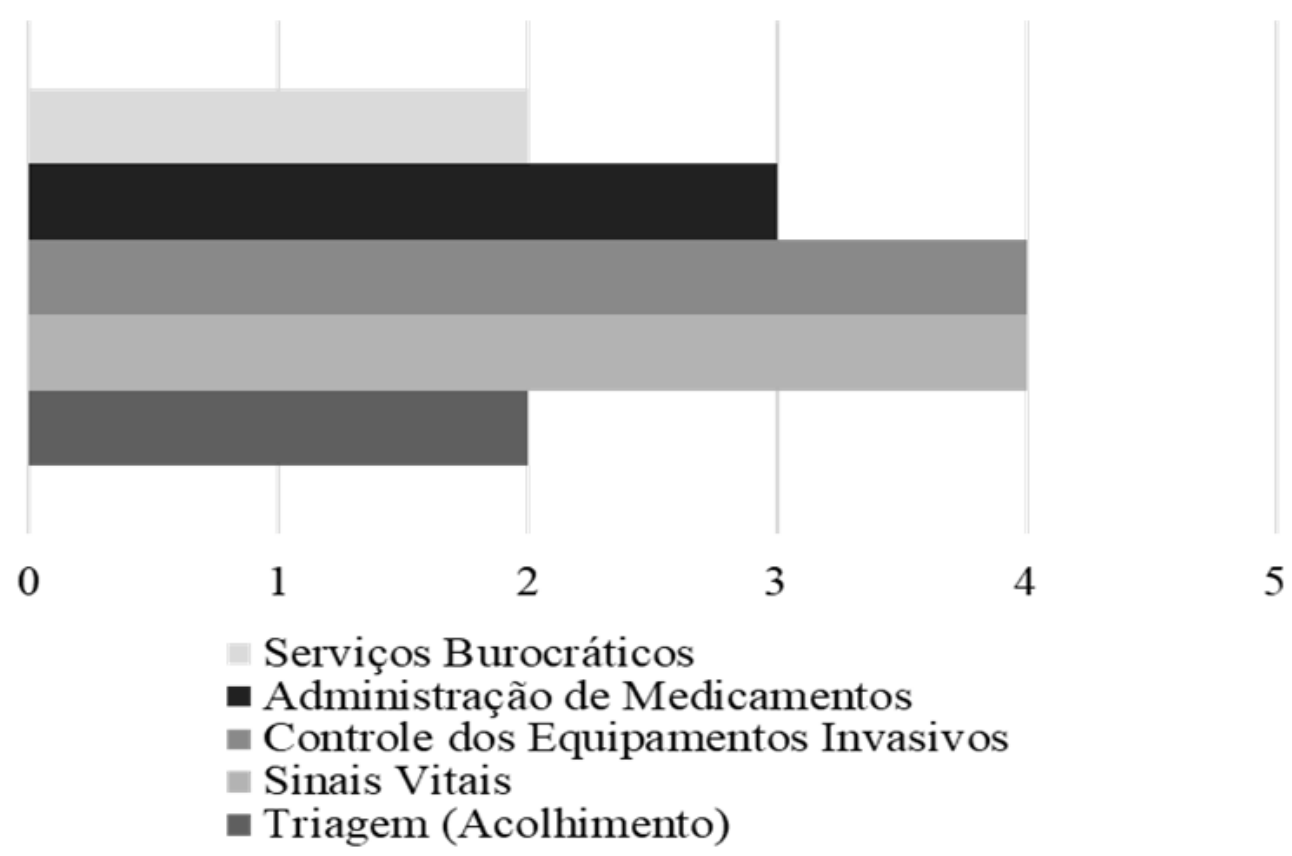

Fonte: dados da pesquisa, 2018 
Denota-se que a DHEG é uma patologia obstétrica que aparece depois da vigésima semana de gestação, sendo mais frequente no terceiro trimestre e estendendo-se até o puerpério. Caracteriza-se por apresentar hipertensão arterial, edema e/ou proteinúria, podendo provocar convulsões, sangramentos e coma ${ }^{5}$.

\section{Discussões}

Tradicionalmente, a gravidez é considerada um evento fisiológico. No entanto, cerca de $20 \%$ das mulheres grávidas desenvolvem patologias obstétricas associadas à mortalidade maternal e perinatal ${ }^{5,8}$. Em todo o mundo, todo ano, meio milhão de mulheres morre durante a gravidez $\mathrm{e}$ parto devido algumas complicações obstétricas. Infelizmente, um número significativo de urgências e emergências obstétricas ocorrem, portanto, prevenção, identificação e intervenção precoce tornam-se fundamentais para evitar distorcias obstétricas 9 .

Diante do exposto e com base nas inferências analisadas percebe-se que os dados deste estudo pontificam empiricamente que o panorama das produções cientificas acerca da assistência de enfermagem as urgências e emergências obstétricas no Brasil são escassas. Nos últimos 10 anos, com base na metodologia adotada, não houve publicações significativas sobre essa temática. No entanto, as publicações existentes revelam que as condutas de enfermagem recaem sobre os protocolos clínicos assistências e sobre as políticas de urgência e emergência, bem como as próprias competências e habilidades da enfermagem.

Essa afirmação pauta-se em estudos ${ }^{9-11}$ que defendem que a assistências as urgências e emergências obstétricas se configuram como a realização da triagem; serviços burocráticos, a monitorização dos sinais vitais e a administração de medicamentos e controle de equipamentos. Esses procedimentos já são de competência do enfermeiro em toda e qualquer assistência humanizada, equânime e resolutiva.

No entanto, é importante esclarecer que os estudos trazem a triagem como sendo sinônimo de acolhimento, o que denota pouca clareza quanto o real sentido da palavra e consequentemente da prática. Acolhimento é, antes de tudo, uma diretriz política e operacional do SUS. É postura ética, uma atitude em relação à garantia de acesso aos serviços de saúde, à qualidade e integralidade da atenção. Para a Política Nacional de Humanização ${ }^{12}$, acolhecimento

[...] traduz-se em recepção do usuário nos serviços de saúde, desde a sua chegada, responsabilizandose integralmente por ele, ouvindo sua queixa, permitindo que ele expresse suas preocupações. Implica prestar um atendimento com resolutividade e responsabilização, orientando, quando for o caso, o paciente e a família, garantindo a articulação com os outros serviços de saúde para a continuidade da assistência quando necessário ${ }^{12}$.

Dentro deste sentido de acolhimento, existe ainda o Acolhimento com Classificação de Risco (ACCR), compreendido também, como triagem. Este é um dispositivo técnico-assistencial que permite, além da garantia de acesso, concretizar o princípio da equidade, pois possibilita a identificação das prioridades para atendimento, ou seja, atender segundo a necessidade de saúde/ gravidade/ risco ou vulnerabilidade de cada usuário. Avaliar riscos e vulnerabilidade implica estar atento tanto ao grau de sofrimento físico quanto psíquico, pois muitas vezes o usuário que chega andando, sem sinais visíveis de problemas físicos, mas muito angustiado, pode estar mais necessitado de atendimento e com maior grau de risco e vulnerabilidade ${ }^{12}$.

Nesta ótica, triagem é uma separação, escolha, seleção, ou seja, um funcionário da unidade ouve a queixa do paciente e seleciona para qual profissional da unidade ele irá encaminhá-lo enquanto que acolhimento é a humanização dos serviços de saúde.

Com relaçãoaosserviçosburocrático, percebeuse que os profissionais da enfermagem inferem passarem mais tempo realizando protocolos de atendimentos emergenciais no papel, do que realizando a conduta assistencial propriamente dita. Com relação aos sinais vitais, administração de medicamentos e controle de equipamentos, 
alguns estudos apontaram que a frequências das mulheres nas urgências e emergências recaem também na insegurança ao sentirem alguns sinais e sintomas típicos da gravidez. Há um desconhecimento, mitos que rodeiam o período da gestação, do parto e do nascimento levando na maioria das vezes, à insegurança e à preocupação da mulher e dos acompanhantes. Mesmo sendo acompanhada durante o pré-natal muitas vezes a falta de informação clara e objetiva faz com que procure serviços de urgência e maternidade com frequência, assim é de fundamental importância que haja a construção de um vínculo com os profissionais e os serviços de saúde principalmente o momento do parto.

Outro achado, não menos importante, referese ao atendimento das gestantes nos postos de urgência e emergência. Evidenciou-se que a maior parte do atendimento de mulheres gestantes, se referem a pré-eclâmpsia, a eclâmpsia e a síndrome hipertensiva ocorridos do terceiro ao sétimo mês gestacional. No tocante aos fatores relacionados à intercorrências obstétricas, houve destaque para fatores individuais como: faixa etária; obesidade; sobrepeso; tabagismo; estresse; consumo de bebida alcoólica; existência de comorbidades e antecedente obstétrico de aborto, hipertensão ou proteinúria. A precária situação socioeconômica e a dificuldade de acesso aos serviços especializados também foram mencionadas. Como consequências das urgências e emergências obstétricas foram indicadas repercussões maternas e fetais reversíveis ou não, tais como: gravidez de risco; trabalho de parto prematuro; abortamento; ansiedade; edema agudo de pulmão; síndrome de HELLP; acidente vascular encefálico; insuficiência renal; convulsão e coma materno; déficit de volume de líquido amniótico; hemorragia cerebral materna; sofrimento fetal e morte materna. Foram apontadas intervenções de enfermagem para a síndrome hipertensiva, pré-eclâmpsia, eclampsia e placenta prévia, mas não foram indicadas intervenções nos casos de hemorragias, aminiorrexe prematura e infecção do trato urinário/pielonefrite $e^{9,13,14}$.

Com estes achados, o presente estudo evidenciou que embora esteja disponível um elevado contingente de publicações acerca do campo obstétrico, há um número reduzido de artigos com enfoque na assistência de enfermagem as urgências e emergências obstétricas. Trata-se, portanto, de uma importante lacuna identificada por esta investigação uma vez que se acredita que as publicações sejam menos frequentes porque esta área em específico tem sido pouco explorada no âmbito da pesquisa. Entretanto, cabe ressaltar que persistem os problemas organizativos dos serviços de urgência e emergência hospitalar que poderiam ser beneficiados com a incorporação de resultados de pesquisa. Para a enfermagem acredita-se que há o desafio de investir esforços para reconfigurar a prática assistencial e gerencial em serviços de urgência e emergência obstétrica, uma vez que a enfermagem necessita de aprimoramento para desenvolver com habilidade e destreza as competências da obstetrícia, contribuindo ativamente para mudar o cenário que se apresenta.

\section{Considerações finais}

Este estudo tinha por objetivo maior descrever a produção científica da enfermagem sobre as urgências e emergências obstétricas, concatenando estes achados em uma revisão bibliométrica. Para tanto foi realizado uma pesquisa incessante na busca de material para compor este estudo, porém, notou-se uma escassez no que tange a temática trabalhada. Todavia, mesmo com tais dificuldades na aquisição de matérias conseguiu-se alcançar o objetivo deste estudo, assim como, tecer reflexões salutares acerca das evidências compiladas nesta pesquisa.

Evidenciou-se que os profissionais de enfermagem realizam assistência as urgências e emergências obstétricas ao realizarem a triagem, na monitorização dos sinais vitais, na administração de medicamentos e no controle de equipamentos. Percebeu-se ainda nos estudos que a procura ginecológica voltada para os cuidados obstétricos precisam ser melhor informados durante o pré-natal na atenção primária. Pois os sinais e sintomas das gestantes que procuram os serviços de urgência e emergência, nem sempre caracterizam o atendimento ideal neste nível de atenção. 
No entanto, infere-se que foi possível identificar a assistência de enfermagem, onde está encontrase alicerçada no processo de enfermagem, com destaque na realização da triagem; serviços burocráticos, na monitorização dos sinais vitais, na administração de medicamentos e controle de equipamentos.

Frisa-se que devido a inexistências literária de estudos que desvelem sobre as urgências e emergências obstétricas sugere-se que mais estudos sejam realizados sobre essa temática, uma vez que se torna de extrema necessidade apreender sobre a condução assistencial, perfil profissional e a percepção das gestantes nas nuances que circundam as urgências e emergências obstétricas. Espera-se, portanto, que esse estudo venha contribuir com outras novas pesquisas referentes a essa temática.

\section{Referências}

1. Silva SPC, Prates RCG, Campelo BQA. Parto Normal ou Cesariana? Fatores que influenciam na escolha da gestante. Revista de Enfermagem da Universidade Federal de Santa Maria (REUFSM). 2014;4(1):1-9.

2. Gama AS, Giffin KM, Angulo-Tuesta A, Barbosa GP, D’orsi E. Representações e experiências das mulheres sobre a assistência ao parto vaginal e cesárea em maternidades pública e privada. Cad Saúde Pública. 2009;25(11):2480-8

3. Bittencourt F, Vieira J, Almeida ACCH. Concepção de gestantes sobre o parto cesariano. Cogitare Enfermagem. 2013;18(3):515-20

4. Nascimento E, Hilsendeger B, Neth C, Belaver G, Bertoncello KC. Acolhimento com classificação de risco: avaliação dos profissionais de enfermagem de um serviço de emergência. REE [Internet]. 2011;13(4):597-03.

5. Rezende J. Obstetrícia Fundamental. $10^{\text {a }}$ ed. Rio de Janeiro: Koogan; 2006
6. Silva MG, Marcelino MC, Rodrigues LSP, Toro RC, Shimo AKK. Violência obstétrica na visão de enfermeiros obstetras. Revista da Rede de Enfermagem do Nordeste (Rev Rene). 2014;15(4): 720-728.

7. Araújo EA, Oliveira VC, Silva WAC. Estudo bibliométrico da produção científica sobre a contabilidade gerencial. In: Anais do XII Semead. 2009;22(1):20-31.

8. Medina Gonzalez ME, Nip Fabero OJ, Rodriguez Reyna JC. Acretismo placentario como urgencia en el servicio de obstetricia: Presentación de casos. Revista Médica Electrónica. 2009;31(6):31-42.

9. Nomura RMY, Novoa VANY, Pimenta BSO, Nakamura UM, Moron AF. Validação de questionário para avaliar a vivência e autoconfiança nas emergências na assistência ao parto vaginal. Rev. Bras. Ginecol. Obstet. [Internet] 2014;36(11): 519-524.

10. Santos JKS. Saúde da mulher: motivos de atendimento no serviço de urgência obstétrica às gestantes residentes nas áreas de atuação das ESF no Setor Habitacional do Sol Nascente, Ceilândia - DF, 2014-2015. [Dissertação de Mestrado]. 2016. Brasília: Universidade de Brasília; 2016, $78 \mathrm{p}$.

11. Brilhante AF, Vasconcelos CTM, Bezerra RA, Lima SKM, Castro RCMB, Fernandes AFC. Implementação do protocolo de acolhimento com classificação de risco em uma emergência. Revista da Rede de Enfermagem do Nordeste (Rev Rene). 2016;17(4):569-575.

12. Brasil. Ministério da Saúde. Manual de acolhimento e classificação de risco em obstetrícia. 1.ed. Brasília Ministério da Saúde, 2014.

13. Ataide MM, Santos AAP, Oliveira e Silva JM, Sanches METL. Exame obstétrico realizado pela enfermeira: da teoria à prática. Enfermagem em Foco. 2016;7(2):67-71

14. Oyarzun EE, Kusanovic JP. Urgencias en obstetricia. Revista Médica Clínica Las Condes.2011;22(3):316-331.

\section{Como citar este artigo:}

Matoso LML, Lima VA. Assistência de enfermagem em urgência e emergência obstetríca: um estudo bibliométrico. Rev. Aten. Saúde. 2019; 17(61): 65-73. 